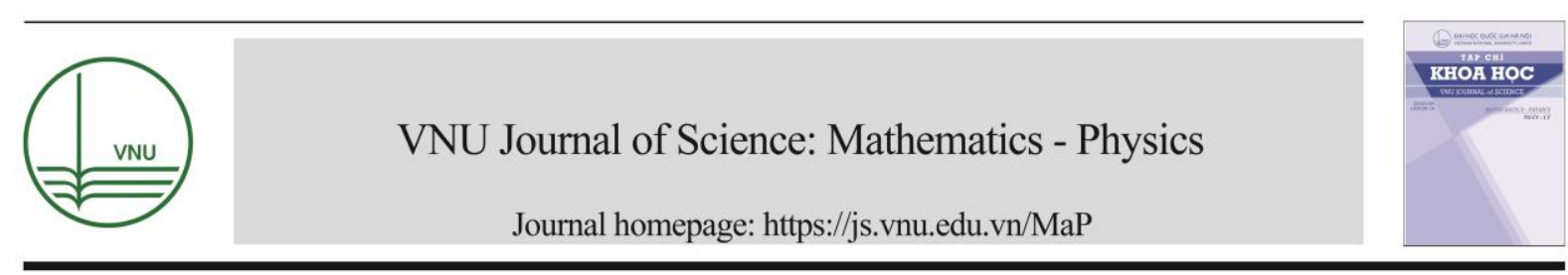

\title{
Perfecting the Advanced 2D Multi-electrode Electrical Exploration Method
}

\author{
Vu Duc Minh ${ }^{1, *}$, Do Anh Chung ${ }^{1,2}$ \\ ${ }^{l}$ VNU University of Science, 334 Nguyen Trai, Hanoi, Vietnam \\ ${ }^{2}$ Institute for Ecology and Works Protection, Vietnam Academy for Water Resources, \\ 171 Tay Son, Dong Da, Hanoi, Vietnam
}

Received 26 September 2018, Accepted 30 September 2018

\begin{abstract}
The Advanced Multi-electrode Electrical Sounding method (AMES) proposed by us was introduced at VNU. Journal of Mathematics-Physics, No. 31 (3), 2015. However, the published results are mostly focused on the symmetric MC array and tested on a theoretical model. In this paper, the authors continue systematically presenting and supplementing the results obtained after the study, namely the finalization and renaming of the AMES method into the Advanced 2D Multi-electrode Electrical Exploration (AMEE) method, including MC array, data collecting and processing, and testing on theoretical model and practical application to confirm the superiority of this method.
\end{abstract}

Keywords: MC array, Control File, Advanced 2D Multi-electrode Exploration.

\section{Background}

As we all know, with the aim of increasing the efficiency of constant-current electrical exploration methods in general, constant-current electrical sounding methods in particular, a system of Improved Electrical Sounding methods (IES) was successfully proposed in $2001[1,2]$.

The automatic multi-electrode array along the line and multi-channel multi-electrode automatic array allows the traditional quad-electrode array to be more flexible with more continuous data collecting to improve productivity as well as quality and reliability of the information obtained. Therefore, the Improved 1D Electrical Sounding method is rapidly replaced by the Multi-electrode Electrical Exploration (MEE) method, especially in complex environmental conditions. Continuing researching on the improvement of the method when combining the 2D Multi-electrode Electrical Exploration method with IES methods to take advantage of all the strengths of those methods,

\footnotetext{
*Corresponding author. Tel.: 84-914658586.

Email: minhvd@vnu.edu.vn

https//doi.org/ 10.25073/2588-1124/vnumap.4291
} 
resulting in the Improved Multi-electrode Electrical Exploration method, now called Improved 1D Multi-electrode Electrical Sounding (IMES) launched in 2010 [3]. However, it is only limited to 1D surveying.

In order to make optimal use of the existing equipment and for the Multi-electrode Electrical Exploration to be more effective in environmental research, the research into the development and integration of the advantages of the MEE/IMES and IES methods was continued, resulting in the creation of the Advanced Multi-electrode Electrical Sounding (AMES) method by 2015 [4]. However, the published results for the AMES method are only mainly focused on the advanced symmetrical multi-electrode array (now accurately called advanced symmetrical 2D multi-electrode array - in short, symmetrical MC array) tested on a theoretical model. Completing the AMES method is indispensable, so the authors have continued studying, and systematically presenting and supplementing the results obtained in this paper, which is the finalization and renaming of the AMES method into the Advanced 2D Multi-electrode Electrical Exploration method, including MC array, data collecting and processing, and testing on theoretical model and practical application to confirm the superiority of this method.

\section{Perfecting the advanced 2D multi-electrode array}

IES methods have been proposed with the use of two improved arrays: i) Improved symmetrical array (KM01) and ii) improved dipole array (KM02).

To improve the AMEE method, the MC array has to be finalized first. In the paper [4], we showed the principles of building MC array and examples on symmetrical MC array.

For example, with the symmetrical MC array below:

$\mathrm{MA}=\mathrm{AB}=\mathrm{BN}=$ na.

$\mathrm{MA}=4 \mathrm{AB}=\mathrm{BN}=4 \mathrm{na}$ (extend receive)

$\mathrm{MA}=\mathrm{AB}=\mathrm{BN}=3$ na (extend transmit)

in which: $\quad \mathrm{a}$ - the distance between measurement electrodes;

$\mathrm{n}-$ the $\mathrm{n}^{\text {th }}$ measurement.

Thus, the symmetrical MC multi-electrode array with the distance of first $\mathrm{AB}$ (in the position 27 and 28), we will have 2 measurement times with $2 \mathrm{MN}$ distances (M1N1 in the position 26, 29 and M2N2 in the position 23, 32) that could be presented as follows:

$\mathrm{M}_{2} \quad \mathrm{M}_{1}$ A B N $\mathrm{N}_{1} \quad \mathrm{~N}_{2}$

$123 \quad 3 \quad 5 \quad 5 \quad 6 \quad 78 \quad 91011121314151617181920212223242526272829303132333435363738394041424344454647484950515253545556$

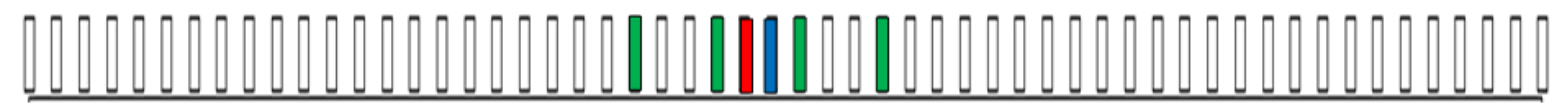

After that, we shall extend AB (in the position 26 and 29), we will get 2 measurement times with 2 MN distances (M1N1 in the position 23, 32 and M2N2 in the position 14, 41) that could be presented as the following diagram:

$\begin{array}{llllll}\mathrm{M}_{2} & \mathrm{M}_{1} & \mathrm{~A} & \mathrm{~B} & \mathrm{~N}_{1} & \mathrm{~N}_{2}\end{array}$

$12234456678 \quad 9 \quad 1011121314151617181920212223242526272829303132333435363738394041424344454647484950515253545556$

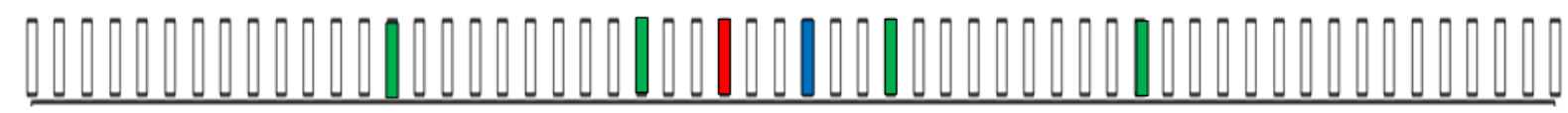


Since the multi-electrode array has the same distance between poles, corresponding to the symmetrialc MC array is the dipole MC array as follows:

$3 \mathrm{AB}=3 \mathrm{BN}=\mathrm{NM}=3 \mathrm{na}$ (right hand side)

$\mathrm{NM}=3 \mathrm{MA}=3 \mathrm{AB}=3 \mathrm{na}$ (left hand side)

in which: $\quad \mathrm{a}-$ the distance between electrodes

$$
\mathrm{n}-\mathrm{the}^{\text {th }} \text { measurement }
$$

For dipole MC array, at each measurement point with the distance of first $\mathrm{AB}$ (e.g. at position 14 and 15), we will have two measurements with the left and right hand sides of $M N\left(M_{T} N_{T}\right.$ at position 10,13 and $\mathrm{M}_{\mathrm{F}} \mathrm{N}_{\mathrm{F}}$ at position 16,19$)$, can be represented as follows:
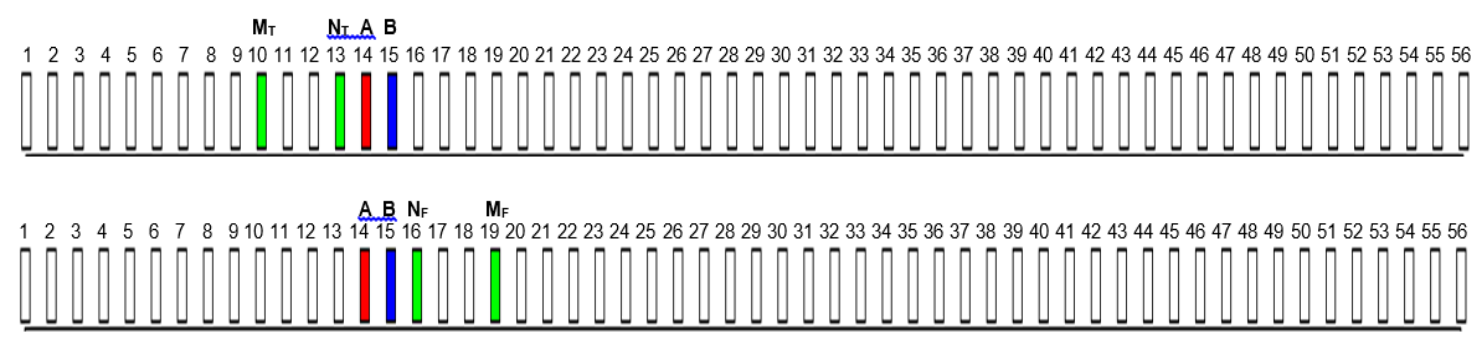

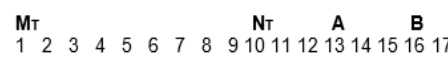

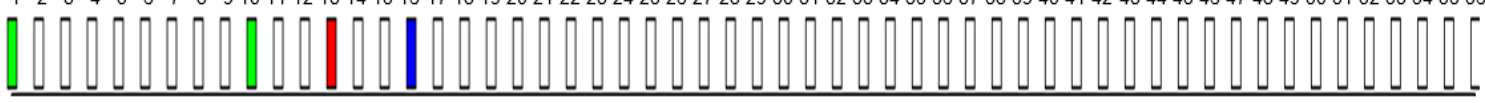

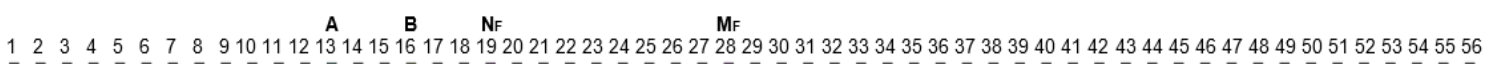

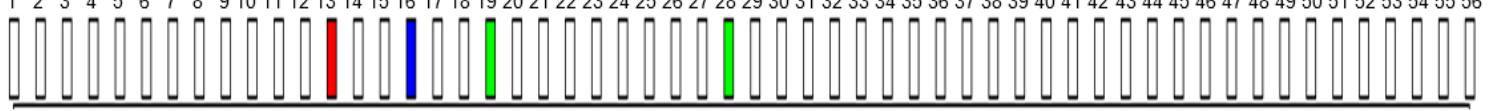

Then extending $A B$ (at position 13 and 16 we will have 2 measurements with $2 \mathrm{MN}$ sides $\left(\mathrm{M}_{\mathrm{T}} \mathrm{N}_{\mathrm{T}}\right.$ at positions 1, 10 and $\mathrm{M}_{\mathrm{F}} \mathrm{N}_{\mathrm{F}}$ at position 19,28), can be expressed as follows:

On this basis, coupling and placement of electrodes shall be done in such a way that there is a 56pole MC array on the principle of coupling of electrodes from the beginning to the end of the line. Thus, the dipole MC array also have all the features of the improved 1D dipole array, the improved 1D multi-electrode array and conventional multi-electrode array.

The following are two examples of electrode layout and data points of the AMEE method.

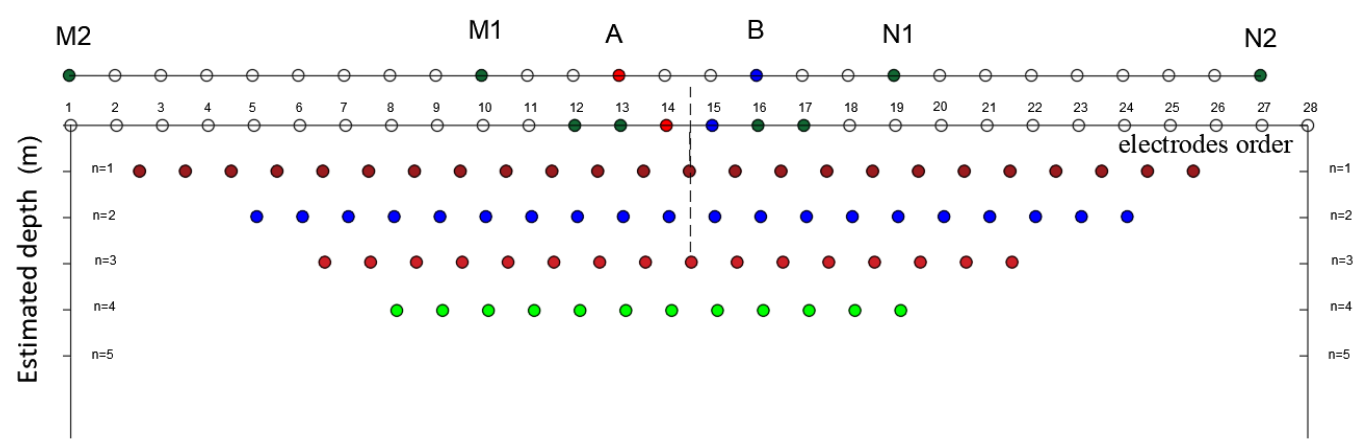

Figure 1. Electrodes layout and data points of the AMEE method with symmetrical MC array for 28 electrodes. 


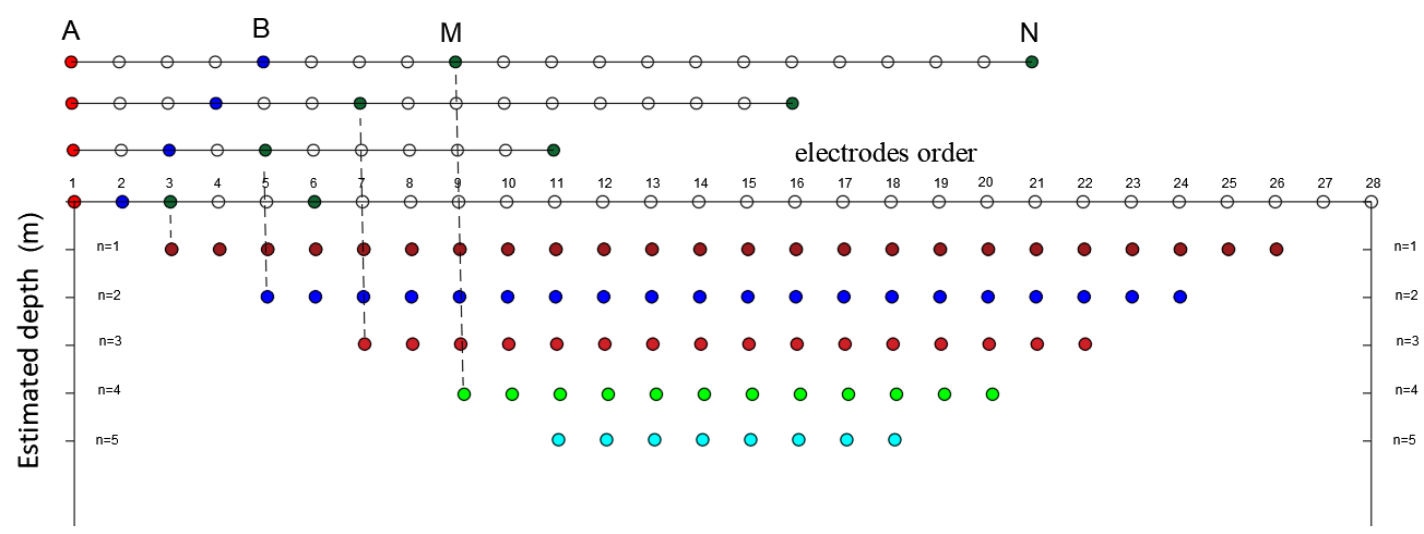

Figure 2. Electrodes layout and data points of the AMEE method with dipole MC array for 28 electrodes.

\section{Complete the process of surveying, collecting and processing data}

In essence, the survey and data collection process of the AMEE method is similar to that of the IMES method [3]; the only differences are AMEE method uses the MC array (which has a different coefficient), extension of distances between electrodes, the number of values obtained for each fixed $\mathrm{AB}$. It is the basis for building and being shown in the file that controls the automatic measurement of the method.

This article only present contents that supplement, perfect, and need to be paid attention to regarding the process of surveying, collecting and processing data of the AMEE method.

\subsection{Preparation}

\subsubsection{Selecting array and equipment}

Depending on the purpose of the study, the subject that needs to be surveyed as well as the terrain of the site conducting the field work; based on the original information from the readily available materials, we choose the array, parameters as well as equipment accordingly that are highly effective.

AMEE can be used by SuperSting R1 [5] or SuperSting R8/IP [6] by Advanced Geosciences Inc. (US), and correspondingly, the MC array described above.

Before creating the control file, we have to select the array, the number of electrodes and the proper parameters for the array.

\subsubsection{Setting up control file}

The control file determines that the measuring equipment are operating for the purpose of measurement and controls the measurement process. For the MEE method, we had readily available control file provided by the device manufacturer and installed in the equipment. For the IMES method and the AMEE method, control file is manually set up and installed in the equipment before proceeding to the field work.

To create a control file, use the Administrator for SuperSting software with the following steps: Run the Administrator for SuperSting software; Choose SuperSting as the measuring equipment; Enter the number of electrodes used; Select the array; Enter the parameters of the array (such as maximum depth, etc.); Enter the control file name and save the file. 
Here is an example of a control file for the MC array (56 electrodes) for SuperSting R8/IP of the AMEE method:

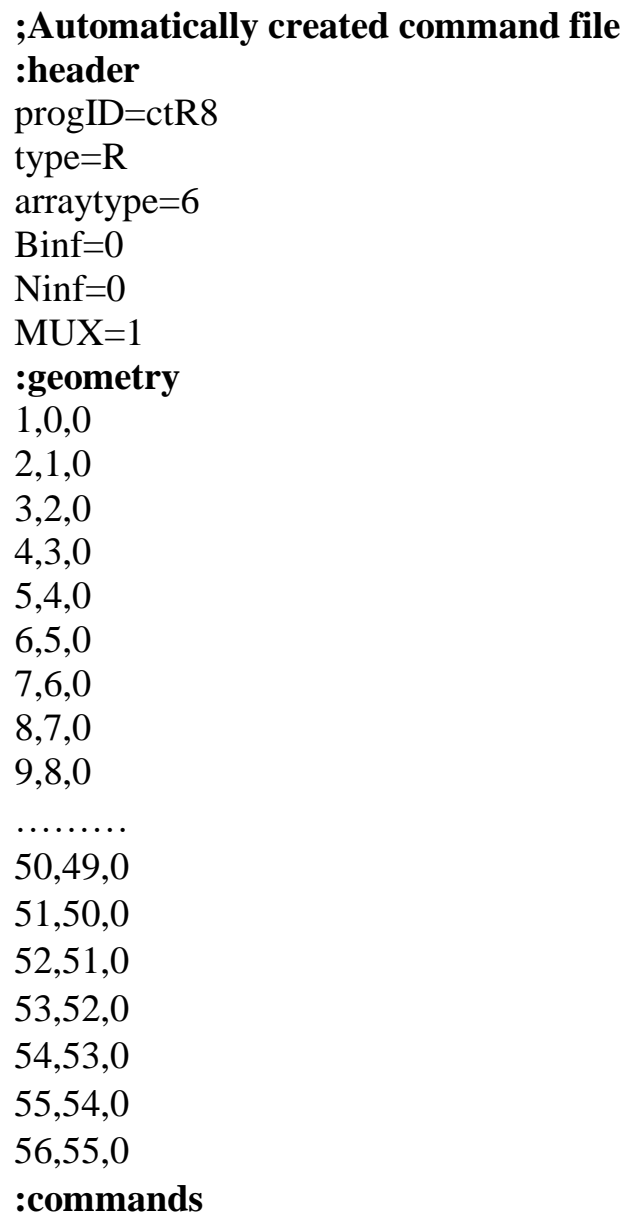




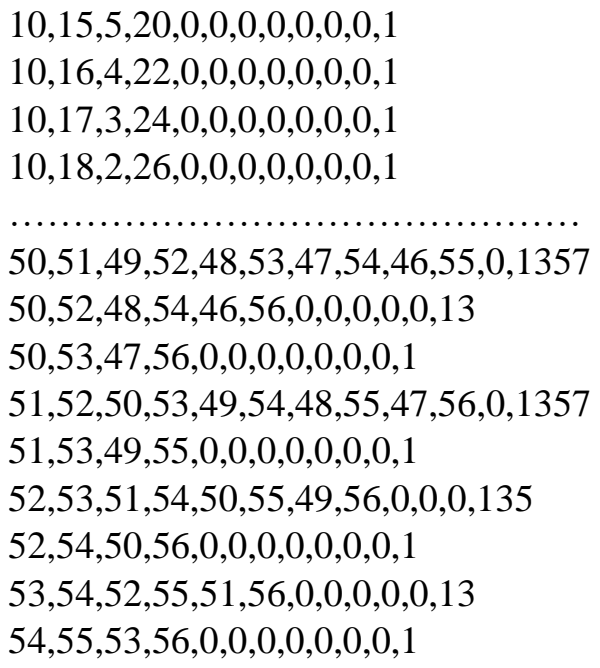

\subsection{Measurement in the field}

Use the SuperSting R8/IP system with 56 electrodes included. Position the electrodes according to the principle of the MC array. The checking and automatic measurement on the network of lines shall be done by the control file automatically set up as described in section 2.1.

Be noted that it is important to use better grounding methods in difficult grounding conditions [7].

\subsection{Data analysis and processing}

From collected data files, proceed in two ways:

- Option 1: Use the program developed by the authors for the AMEE method for analysis and processing.

- Option 2: Use a readily available program such as Res2D [8] or EarthImager 2D [9].

The results are the impedance resistivity geo-electric sections.

\section{AMEE test results with arrays on theoretical models}

In the article [4], the results of the AMEE method were tested with the symmetrical MC array on the theoretical models. In order to further compare the results of the AMEE tested with the MC arrays on the basic theoretical models with the previous ones, proceed as follows: i) Develop theoretical models; ii) Model to traditional multi-electrode arrays (Wenner Schlumbeger and Dipole array) and MC arrays (Symmetric and Dipole); iii) Process the data using EarthImager 2D software; iv) Analyse and evaluate the results.

Here are some test results.

\subsection{Experiment with horizontal model}

The horizontal model is designed to have a thickness of $1 \mathrm{~m}$ (equivalent to the distance between the two nearest electrodes), a width of more than 10 times the thickness and a depth of $1.5 \mathrm{~m}$ (Figure 3 ). 


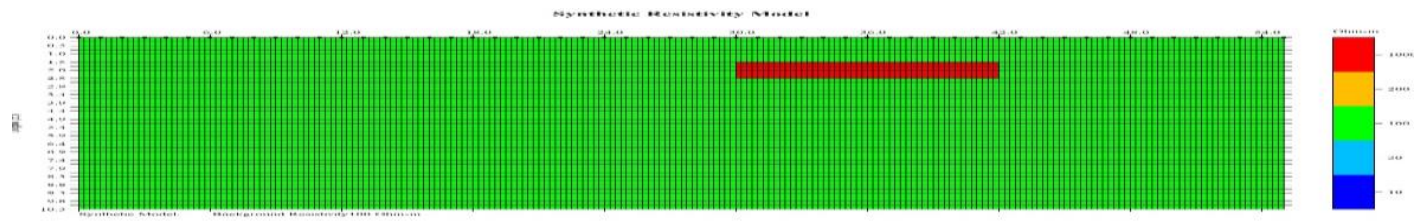

Figure 3. Horizontal testing model.

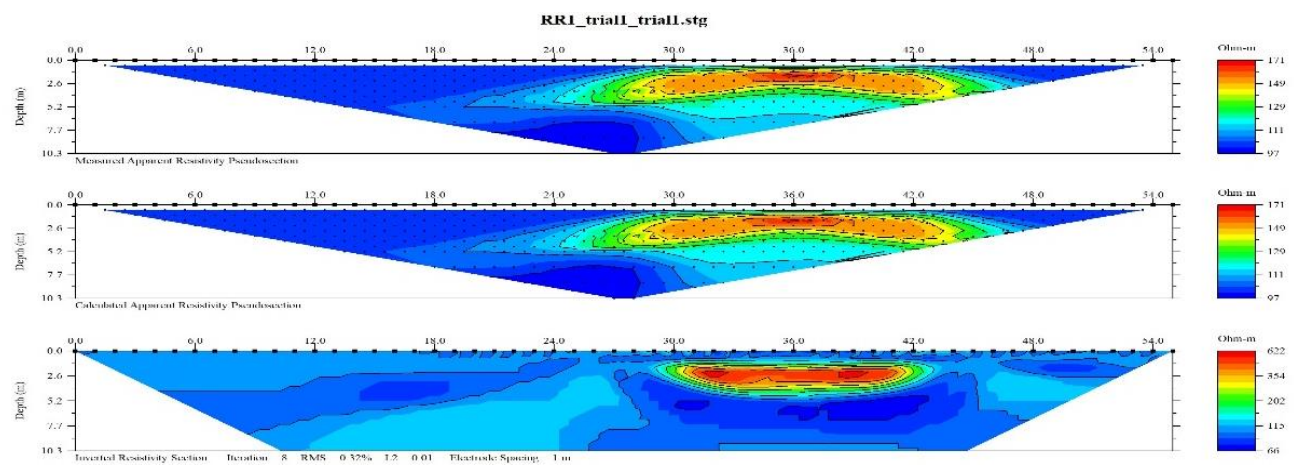

Figure 4. Test results with symmetrical MC array.

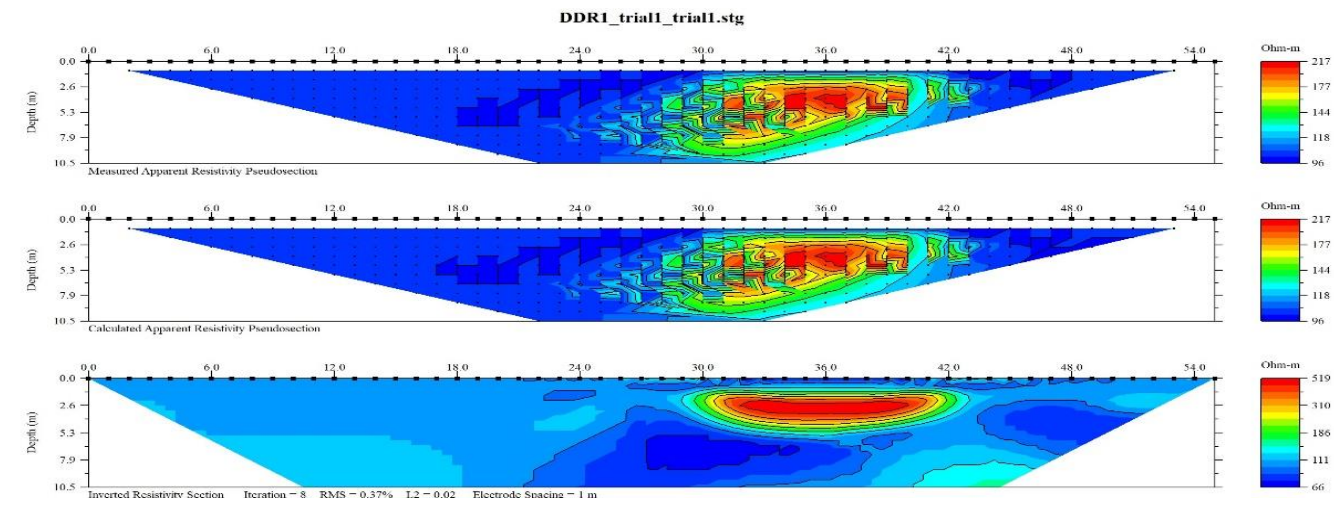

Figure 5. Test results with dipole MC array.
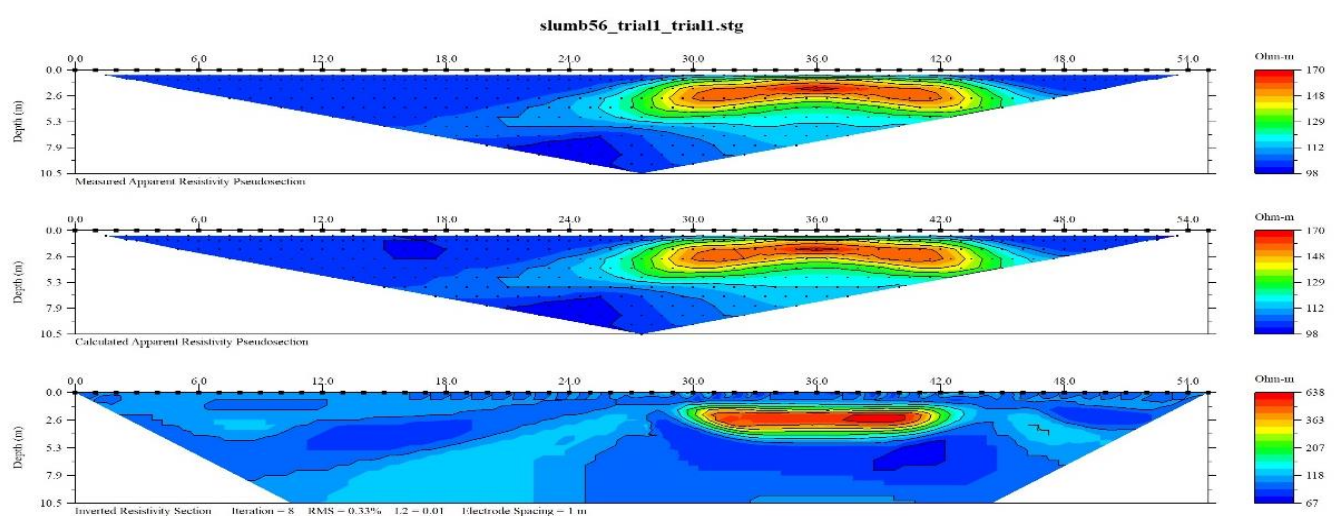

Figure 6. Test results with Wenner Schlumbeger array. 


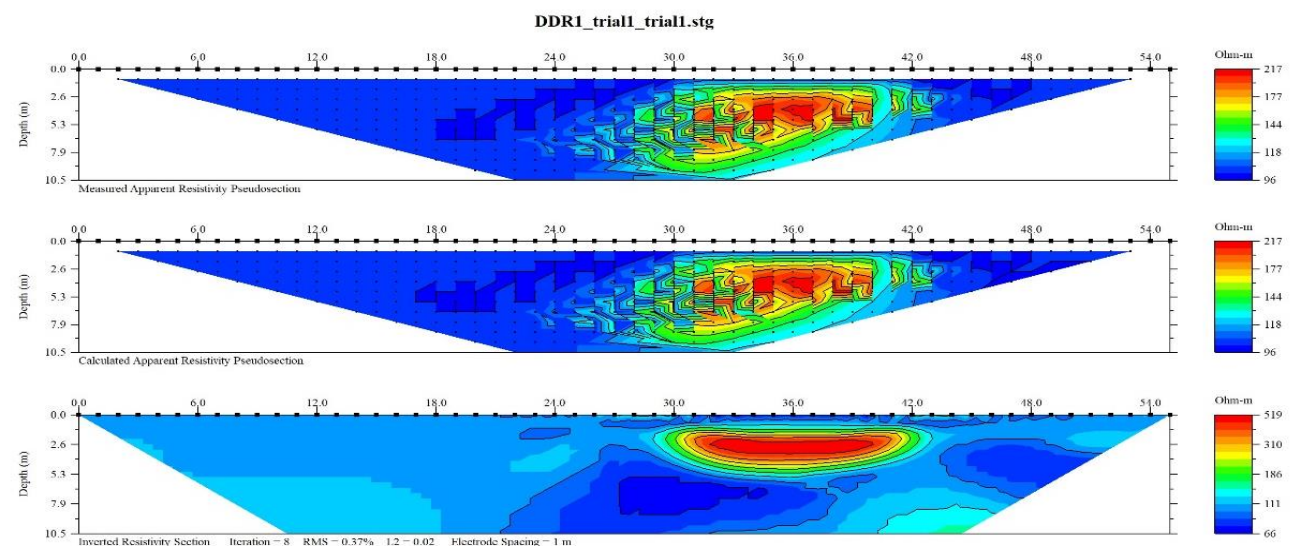

Figure 7. Test results with dipole array.

The test results of the horizontal model in the absence of interference showed that the results on the symmetrical MC array and Wenner Schlumbeger array were the best, with these two arrays indicating exactly the top of the horizontal object. Meanwhile, the dipole MC array and dipole array both have very large variations in depth and thickness. This indicated that the AMEE method with the symmetrical MC array gave the same result as the Wenner Schlumbeger array. Meanwhile, the dipole $\mathrm{MC}$ array and dipole array are not suitable for surveying horizontal objects.

\subsection{Experiment with the inclined model}

The inclined model is designed to be $1 \mathrm{~m}$ wide and tilted at a 45 degree angle to a depth of $7 \mathrm{~m}$ (Figure 8).

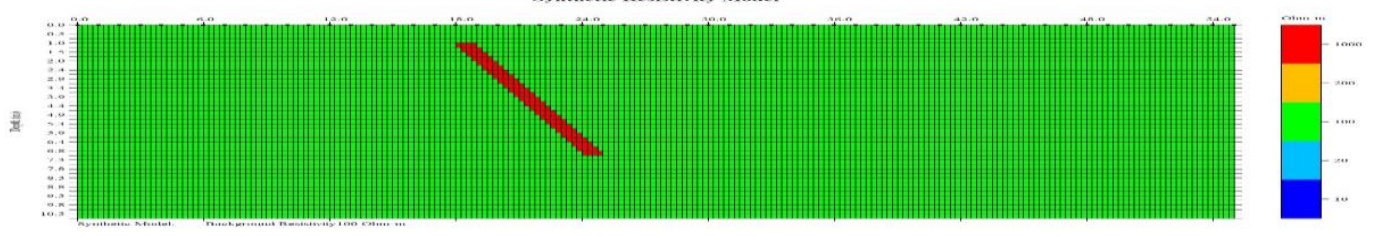

Figure 8. Inclined testing model.

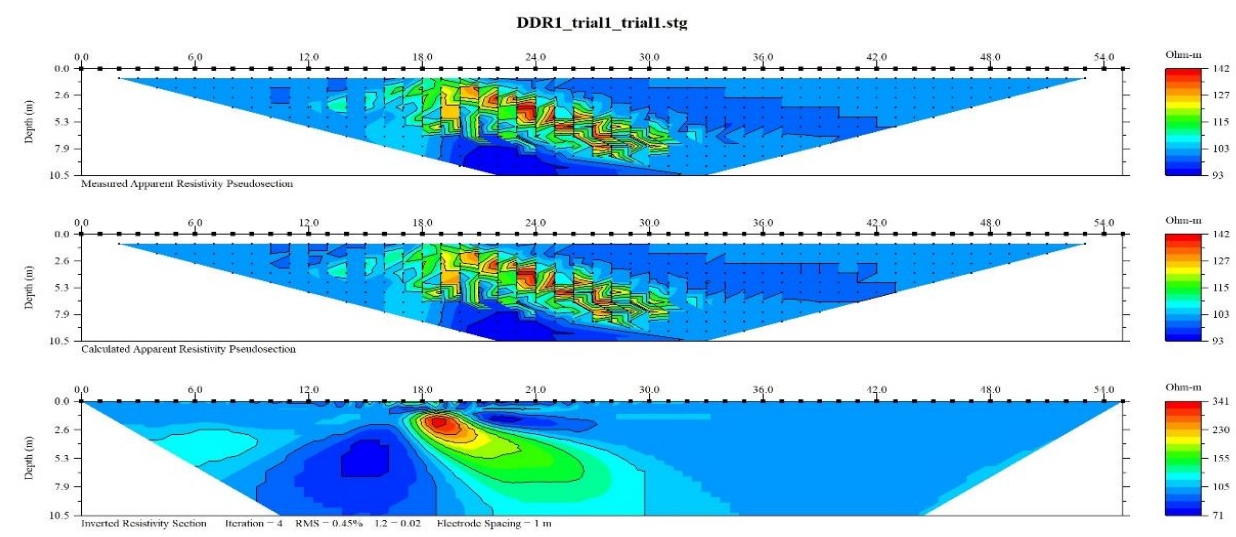

Figure 9. Test results with dipole MC array. 


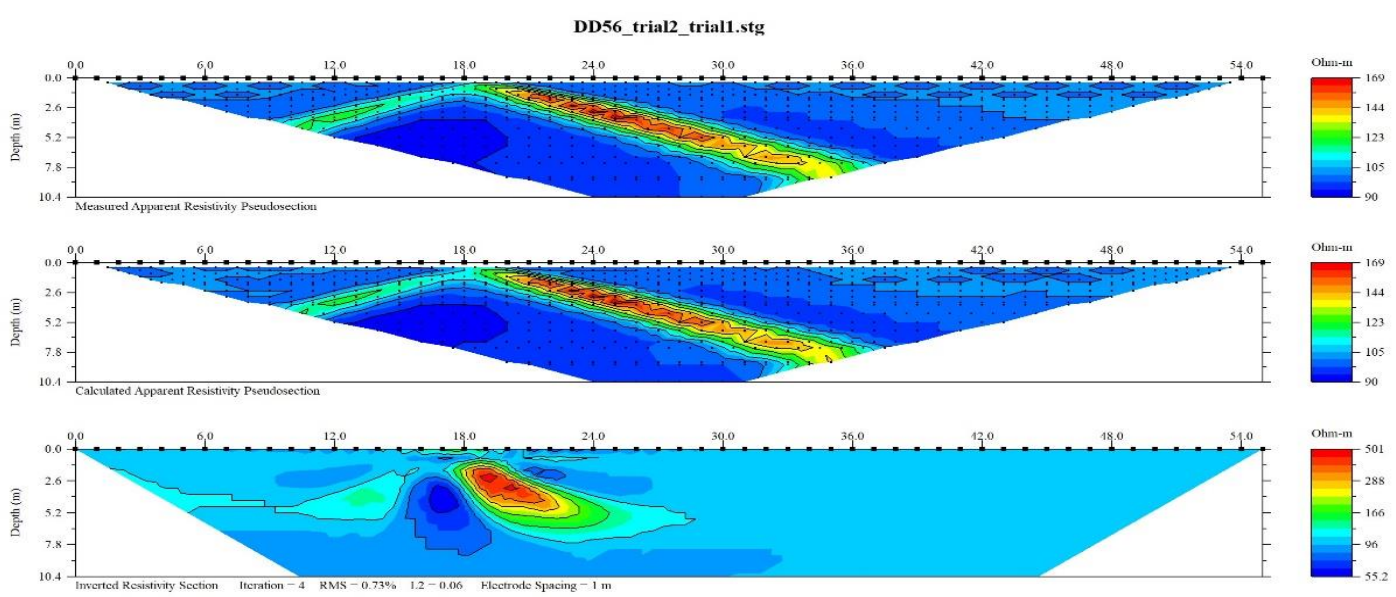

Figure 10. Test results with dipole array.

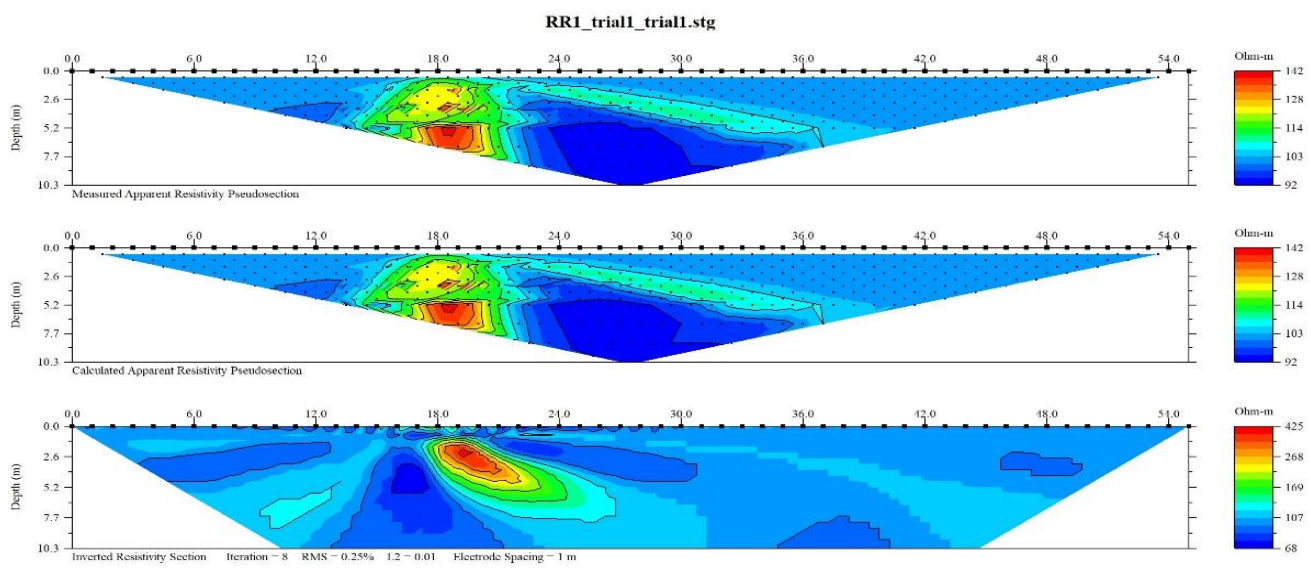

Figure 11. Test results with symmetrical MC array.
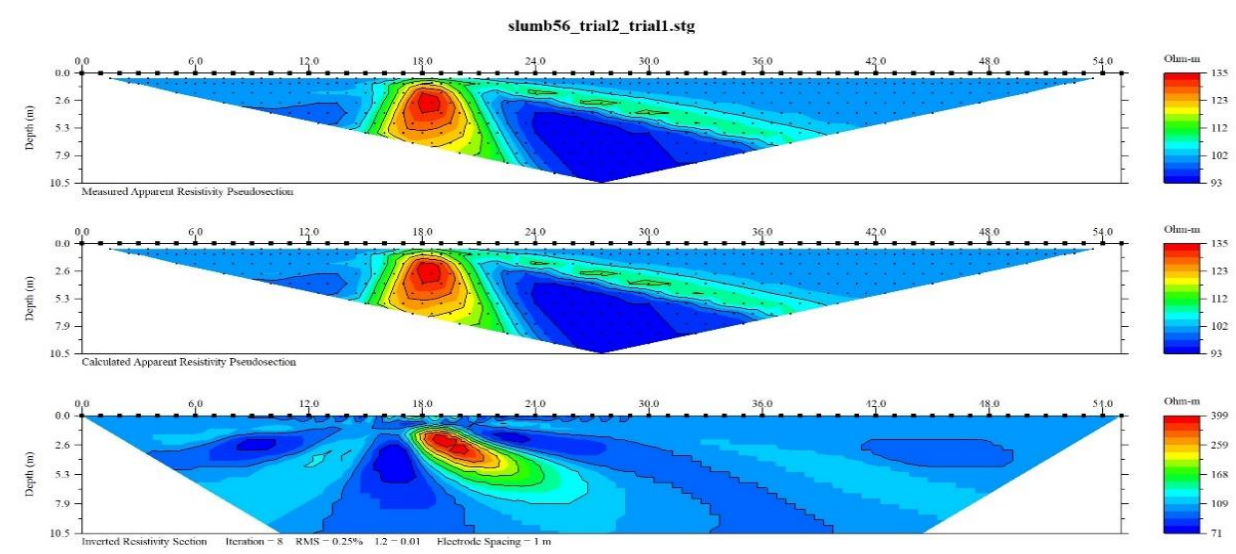

Figure 12. Test results with Wenner Schlumbeger array. 
Test results of the inclined model at a 45 degree angle in the absence of interference showed that all four types of arrays correctly determine the object and angle of the object. However, with the symmetrical MC and Wenner Schlumbeger array, there are many low resistivity anomalies, so it is very difficult to accurately analyze the object. Meanwhile, with dipole MC and dipole arrays, both object and no virtual anomalies were found in the survey line. For inclined model, the dipole MC array gave the same result as the dipole array, but the dipole MC array has a very large gap between collectors and a small array coefficient, hence bigger potential obtained compared to traditional dipole array with smaller margin of error.

\subsection{Experiment with the mixed model}

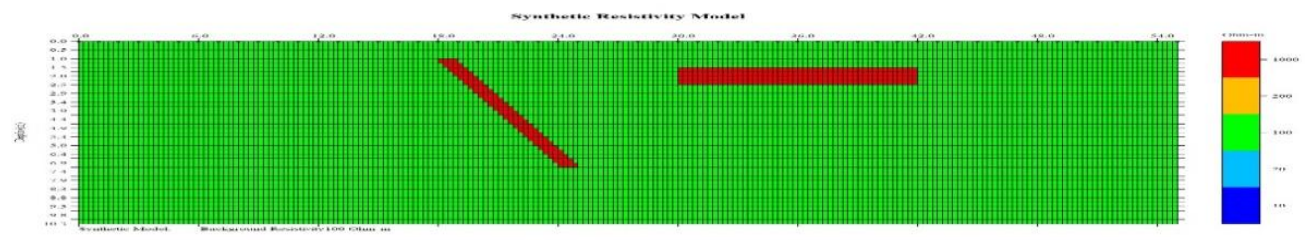

Figure 13. Mixed test model.

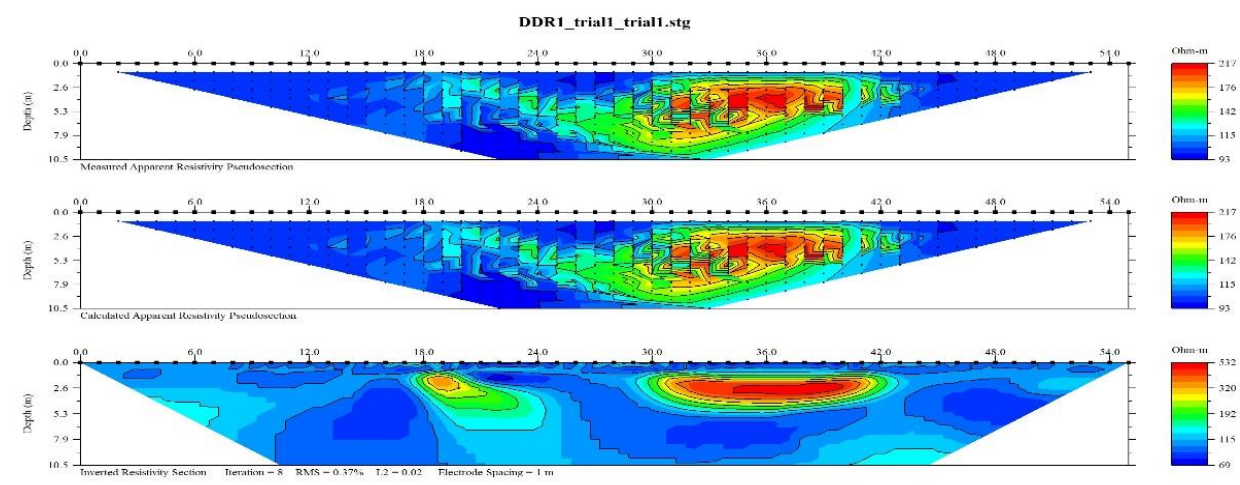

Figure 14. Test results with dipole MC array.

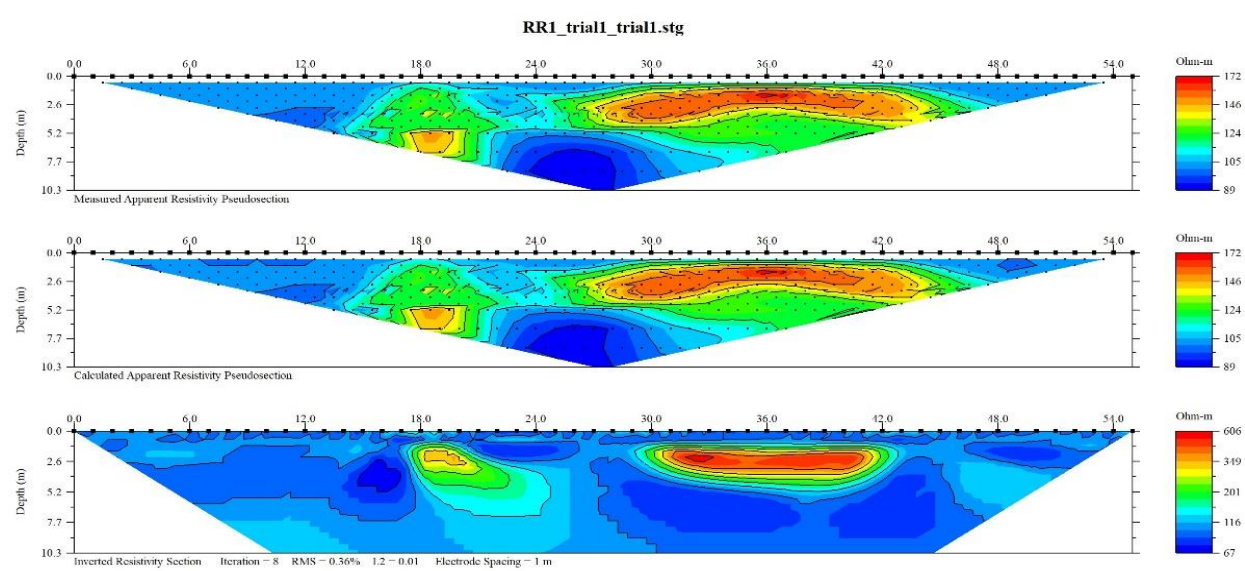

Figure 15. Test results with symmetrical MC array. 


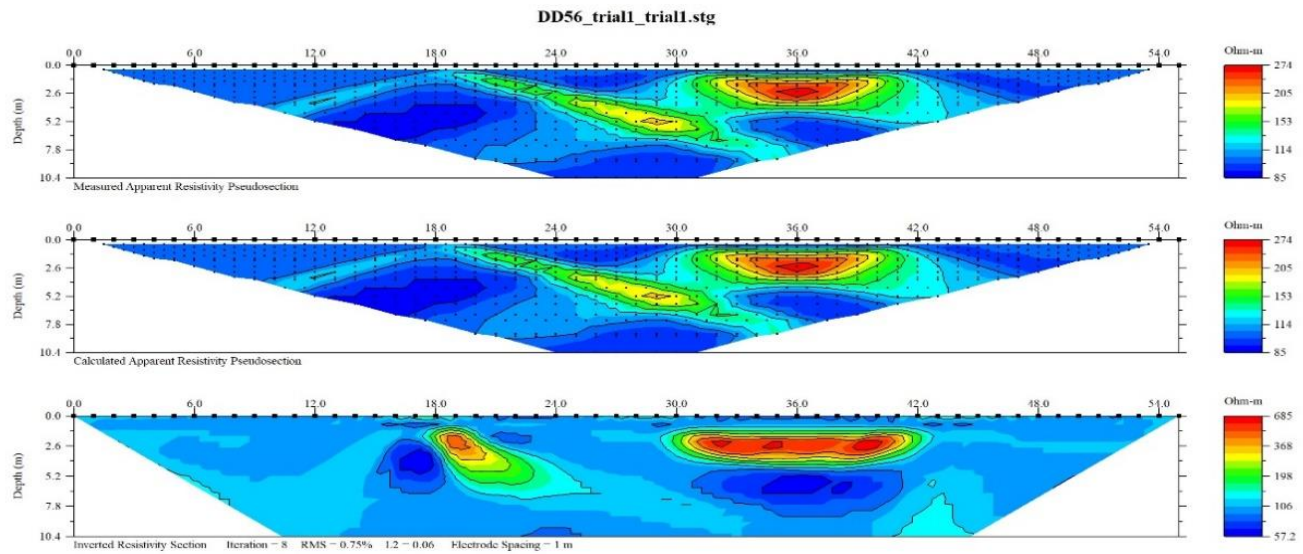

Figure 16. Test results with dipole array
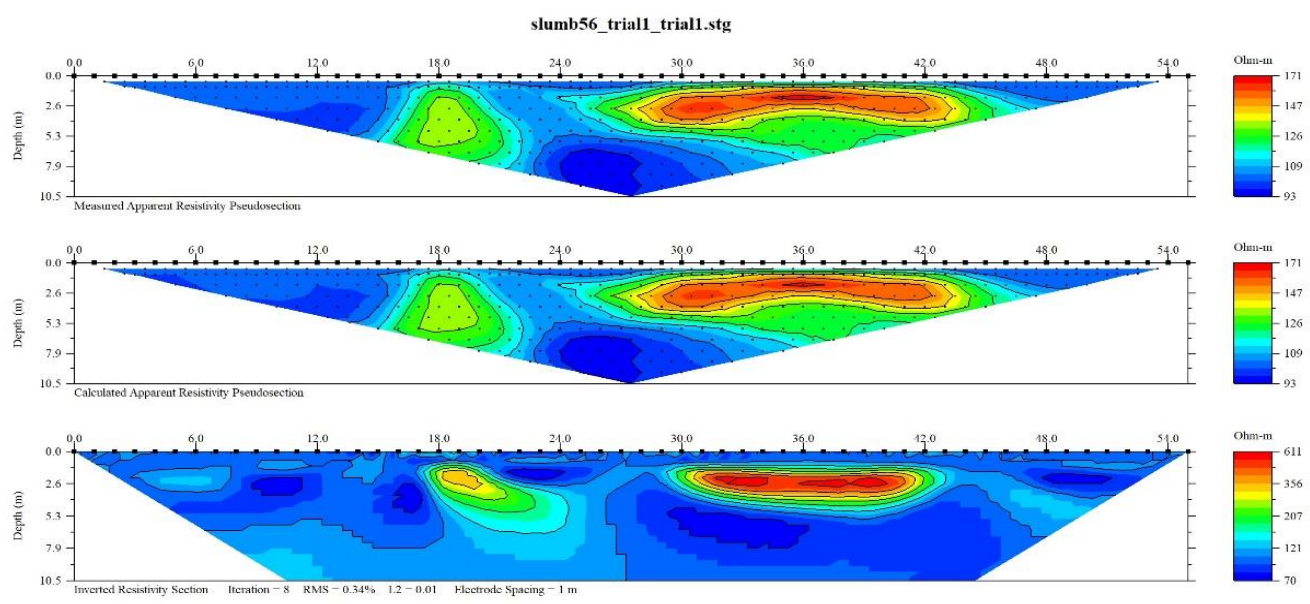

Figure 17. Test results with Wenner Schlumbeger array

The test results of the mixed model in the absence of interference showed that all four types of arrays gave the same results.

\section{Test results on the actual environment}

\subsection{With symmetrical MC array}

To test the applicability of the AMEE method, the Sen Chieu - Son Tay - Hanoi dyke is one of the key routes of the Huu Hong dyke.

A survey was conducted in the downstream section of Sen Chieu dyke from K32+322 to K32+512 by AMEE method with symmetrical MC array and MEE method with Wenner array for comparison. Hereafter, one of the results obtained on T3 (Figure 18) will be presented below. 


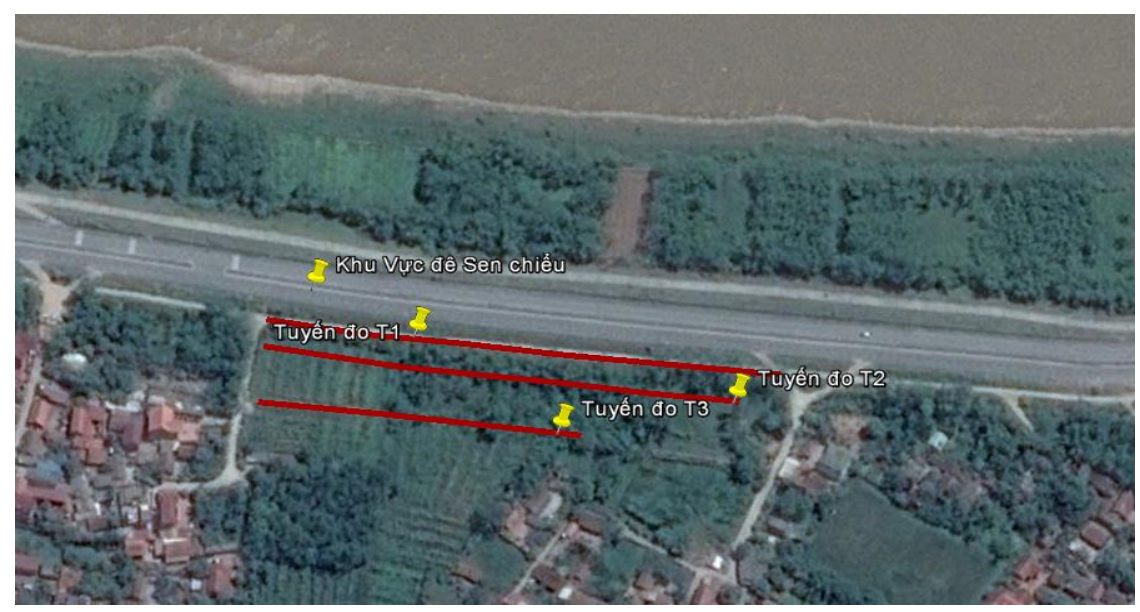

Figure 18. Map of survey route downstream of Sen Chieu dyke (K32+322 $\div \mathrm{K} 32+512)$.

After performing a symmetrical MC measurement, continue to measure using Wenner array. When processing the data, choose the same parameters. The results are as follows:

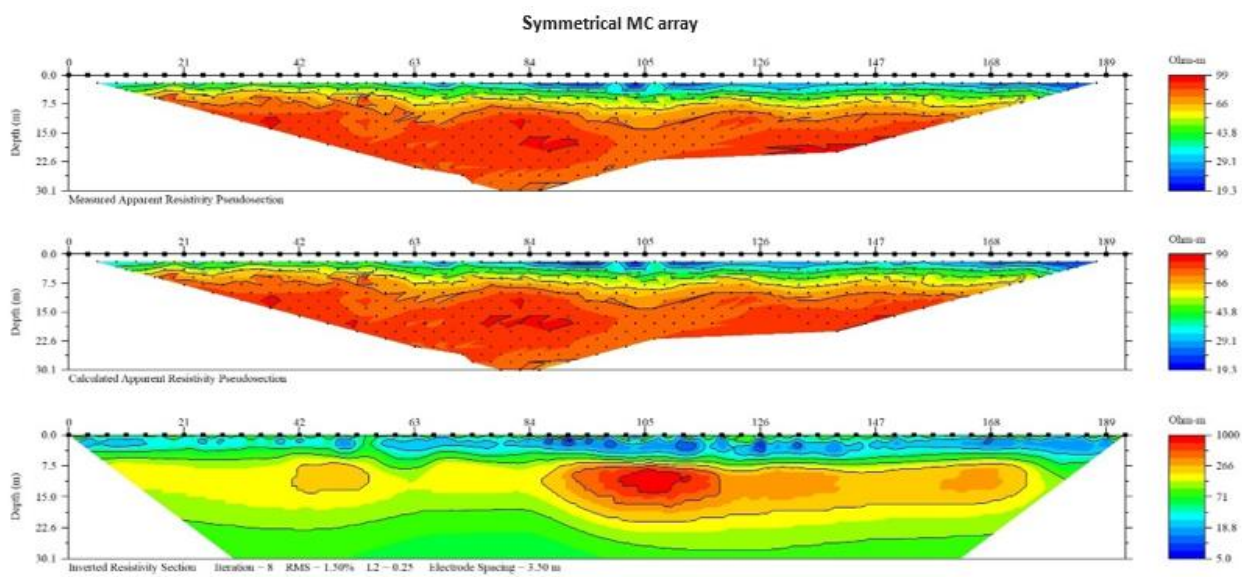

Figure 19. Survey results using symmetrical MC array.

The survey results using the Wenner array and the symmetrical $\mathrm{MC}$ array were similar; in particular, the high resistivity layer i.e. the sand layer has the same depth. However, the results obtained from the symmetrical MC array may allow for more detailed analysis of the anomalies (there is a high resistivity anomaly which, when measured with the Wenner array, is not available); the possible causes are: i) With the Wenner array, $\mathrm{MN}$ is only allowed to open at most no larger than $\mathrm{AB} / 3$, so the signal is large with low level of interference; so should $\mathrm{MN}$ is further opened, the signal is better but averaged, thus not being able to show small anomolies. ii) With the symmetrical MC array, in addition to the same first time measurement as the Wenner array, the MN is extended like the Wenner array, but for each $\mathrm{MN}$ there are two values of $\mathrm{AB}$ (repeated measurement), which allows for more accurate data obtained and identify more detailed objects.

With test results at $\mathrm{K} 32+322-\mathrm{K} 32+512$ in Huu Hong dyke, it is entirely possible to use a symmetrical MC array to determine the permeability areas in the base effectively. 


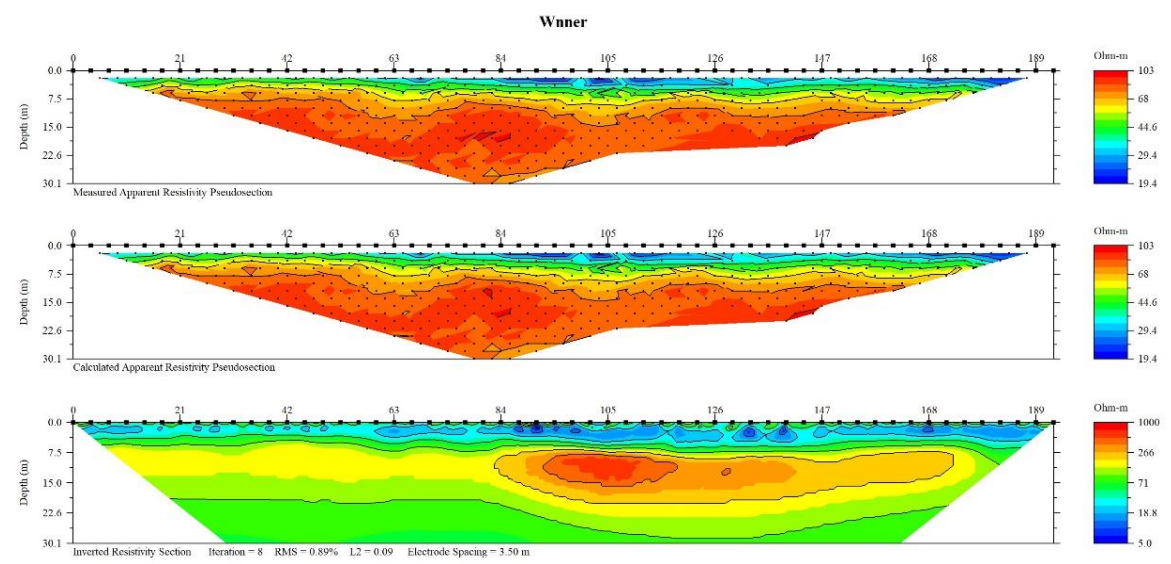

Figure 20. Survey results using Wenner array.

\subsection{With dipole MC array}

The dyke from K41+500-K44+500 Huu Duong is located in Gia Binh district, Bac Ninh province. On the surface of the dyke is poured concrete to make the road. This is the area chosen to test the AMEE method with the dipole MC array and the MEE method with the dipole array to compare and identify cracks in the dike. The results in route $\mathrm{K} 43+115$ are as follows:

K43+115

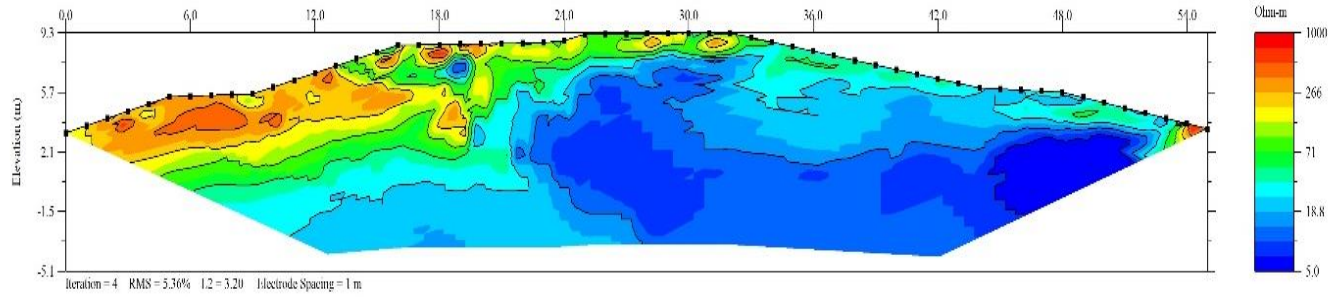

Figure 21. Survey results by dipole MC array.

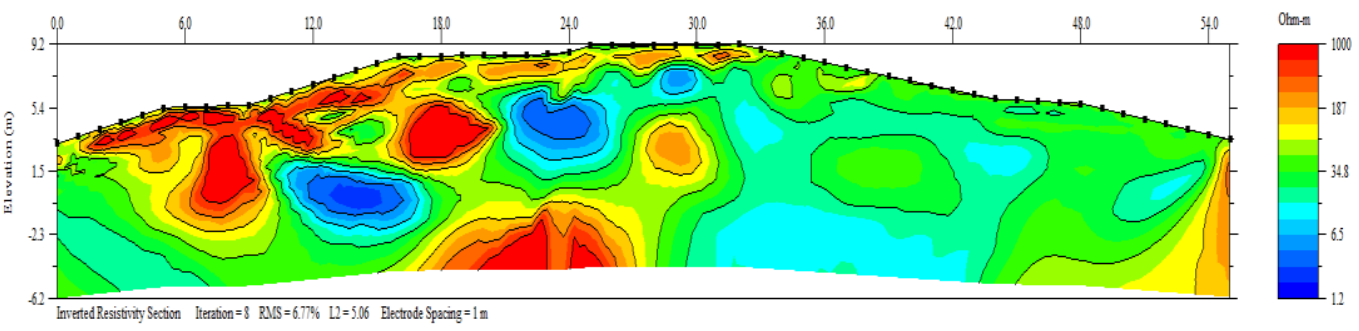

Figure 22. Survey results by dipole array.

The survey results show that when using the dipole MC array, apart from the detection of inclined objects (cracks), horizontal geological layer and the lateral superstructure can still be identified. Whereas the use of dipole array for surveying did not indicate geological layers under the dyke and 
did not indicate a structural layer. The main reason may be: the dipole array has a very large $\mathrm{K}$ coeficient so the potential is small, while the contact conditions on the concrete road is very difficult so the intereference is high; whereas the dipole MC array has small $\mathrm{K}$ coefficient, hence big potential, which avoids random interference due to contacts.

The test results show that the use of dipole MC array is more feasible and superior to the conventional dipole array in the field survey.

\section{Conclusion}

Throughout the research process, the advanced 2D multi-electrode electrical exploration method has been perfected in all stages: complete construction of MC array (symmetric and dipole); improve the process of survey design, data collection and processing.

The AMEE method has been tested on theoretical model and applied testing in practice for very reliable results.

In addition to the detailed conclusions set out above, the general conclusion is that using the AMEE method is more effective than the IES, IMES, and traditional methods because it gives the overall picture of the detailed environment. In addition to clearer detection of the objects, it can also help find other objects undetected by previous methods.

From the above results and comments, we can confirm that the AMEE methodology has integrated and developed all the advantages of the IES, IMES and MEE methods. This is a new method that is superior to older methods and be added to Geophysical Methods in general and to Electricity Exploration in particular.

We will continue publishing the results of practical application of the AMEE method in environmental studies in subsequent papers to demonstrate the potential, effectiveness and advantages of this method.

\section{References}

[1] Lê Viết Dư Khương, Vũ Đức Minh, Các phương pháp mới trong đo sâu điện trở dùng tổ hợp hệ cực đo hợp lý, Tạp chí Các Khoa học về Trái đất, 23(3), 2001, 217.

[2] Vu Duc Minh, Induced-Polarization Sounding methods in a new manner, Journal of Geology, Series B, No. 17 $18,2001,94$.

[3] Vũ Đức Minh, Phương pháp Thăm dò điện đa cực cải tiến, Tạp chí khoa học Đại học Quốc gia Hà Nội, Khoa học Tự nhiên và Công nghệ, 26(4), 2010, 233-241.

[4] Vu Duc Minh, Do Anh Chung, Introduction to the Advanced Multi-electrode Electrical Sounding method, VNU. Journal of Mathematics-Physics, No. 31(3), 2015, p. 1-14.

[5] Advanced Geoscienes, Inc., The SuperSting ${ }^{\mathrm{TM}}$ with Swift ${ }^{\mathrm{TM}}$ automatic resistivity and IP system Instruction Manual, Austin, Taxas, 2000-2009.

[6] Advanced Geosciences, Inc., Instruction manual for SuperSting ${ }^{\mathrm{TM}}$ Earth Resistivity, IP \& SP System with Wi$\mathrm{Fi}^{\circledR}$, Austin, Texas 78726, USA, 2013.

[7] Đỗ Anh Chung, Vũ Đức Minh, Nghiên cứu cải thiện khả năng tiếp đất của các điện cực trong phương pháp điện đa cực cho các môi trường khó tiếp đất, Tạp chí khoa học Đại học Quốc gia Hà Nội, Khoa học Tự nhiên và Công nghệ, 29(2), 2013, 57-69.

[8] Res2dinv Manual Ver.3.5.4, Geotomo Software, Penang, Malaysia, 2004.

[9] Advanced Geosciences, Inc., Instruction manual for EarthImager 2D, Version 2.4.0, Resistivity and IP Inversion Software, Austin, Texas 78726, USA, 2002-2009. 